\title{
El perfil psicológico de los menores denunciados por violencia filio-parental
}

\author{
Izaskun Ibabe ${ }^{1}$ y Joana Jaureguizar \\ Universidad del País Vasco
}

Manuscrito recibido el 28 de noviembre de 2011 / Publicado el 3 de febrero de 2012

\section{RESUMEN}

El principal objetivo de este estudio era comprobar si los menores infractores que habían sido denunciados por maltrato a sus progenitores presentaban características psicológicas y/o psicopatológicas diferentes a los infractores por otros delitos. Se analizaron 413 expedientes judiciales correspondientes a 103 adolescentes de la Fiscalía de Menores de Bilbao (España). Las características más destacables de los adolescentes con delitos de violencia filio-parental fueron las dificultades escolares y la violencia fuera del hogar, lo que podría corresponderse a un perfil de inadaptación social. Además, los infractores que eran violentos hacia alguno de sus progenitores presentaban un porcentaje mayor de trastornos psicológicos que los infractores por otros delitos, $\mathrm{y}$ entre los trastornos más frecuentes se encontraban aquellos incluidos dentro de la categoría de déficit de atención y comportamiento perturbador.

Palabras clave: violencia filio-parental, violencia familiar, menor infractor, adolescente

\footnotetext{
1 Departamento de Psicología Social y Metodología de las Ciencias del Comportamiento Facultad de Psicología. Universidad del País Vasco Avda. Tolosa 70, 20018-Donostia-San Sebastián E-mail: izaskun.ibabe@ehu.es
} 


\begin{abstract}
The main aim of this study was to determine whether adolescents who had abused their parents had different psychological and psychopathological characteristics in comparison to juveniles charged with other types of offence. The authors analyzed 413 files corresponding to 103 young offenders from the office of the Public Prosecutor for Juveniles in Bilbao (Spain). The most noteworthy characteristics of adolescents with charges of child-to-parent-violence were school difficulties and violence outside the home, which could reflect a social maladjustment profile. In addition, juvenile offenders who were violent toward a parent showed a higher percentage of psychological disorders than those offenders who were not aggressive toward parents, and the most common disorders were those included in the attention-deficit and disruptive behaviour category.
\end{abstract}

Key words: parent abuse, family violence, juvenile offender, adolescent

\title{
Introducción
}

La violencia filio-parental hace referencia a las acciones realizadas por los hijos para causar daño físico, psicológico o financiero a sus padres, con el objetivo de obtener poder y control sobre ellos (Cottrell, 2001). Este tipo de violencia intra-familiar ha ido en aumento en los últimos años según los datos aportados por la Fiscalía General del Estado (Matey, 2011). Así, en 2007 fueron 2.683 padres los que denunciaron a sus hijos mientras que en 2010 sumaron 8.000 casos. Estos datos justificarían la preocupación creciente de psicólogos, psiquiatras, educadores y padres por la pérdida de respeto de los adolescentes a la autoridad, y por las vejaciones constantes que sufren los progenitores (Matey, 2011).

Cottrell y Monk (2004) proponen un modelo ecológico para entender los múltiples factores que pueden estar implicados en la violencia filio-parental. Estos autores adaptaron el modelo explicativo de Dodge y Pettit (2003) para el 
comportamiento antisocial al contexto de la violencia filio-parental, e identificaron factores de riesgo como la desigualdad de género, la influencia de la violencia mostrada por los medios de comunicación (a nivel de macrosistema), la pobreza, el estrés familiar, influencias negativas por parte del entorno social, la falta de apoyo social (a nivel de mesosistema), estilos negativos o ineficientes de educación paternal y conflictos con los padres, problemas de salud mental o drogas por parte de los adolescentes o experiencias tempranas de victimización (a nivel de microsistema).

Entre los múltiples factores que pueden estar implicados en la violencia filioparental, este estudio se centrará exclusivamente en las características psicológicas y problemas de salud mental de los adolescentes denunciados por conductas violentas hacia sus progenitores. Atendiendo al contexto socio-educativo, los estudios previos indican que los hijos que presentan conductas violentas hacia sus padres, se caracterizan por conductas disruptivas en el aula durante la infancia y/o adolescencia, dificultades de aprendizaje en la adolescencia (Howard y Rottem, 2008; Ibabe, Jaureguizar, Díaz, 2007), así como problemas de rendimiento escolar (González-Álvarez, Gesteira, Fernández-Arias y García-Vera, 2010). Además, estos jóvenes tienden a relacionarse con grupos de iguales que también ejercen conductas violentas dentro y fuera de sus hogares (Agnew y Huguley, 1989; Cuervo, Fernández y Rechea, 2008). Este perfil violento no sólo se ha hallado en estudios con muestras clínicas o judiciales; las investigaciones que han analizado la violencia filio-parental en la población general también han hallado que los jóvenes que agreden a sus padres se caracterizan por presentar conductas delincuentes en general (Calvete, Orue y Sampedro, 2011; Jaureguizar e Ibabe, 2012).

Otro aspecto de especial interés sería el consumo de sustancias, que requiere un estudio en profundidad. Hay evidencias empíricas de la relación entre el consumo de alcohol y/o drogas y la violencia filio-parental (Ellickson y McGuigan, 2000; Evans y Warren-Sohlberg, 1988; Ibabe y Jaureguizar, 2011; Pagani, Tremblay, Nagin Zoccolillo, Vitaro y McDuff, 2004, 2009). Por el contrario, Walsh y Krienert (2007) hallaron que en su muestra judicial el comportamiento violento de los jóvenes hacia sus padres habitualmente no se producía bajo los efectos del alcohol o las drogas, ya que un bajo porcentaje de los adolescentes (menos del 4\%) indicó estar bajo la influencia de alcohol o drogas cuando cometió el asalto, lo cual significa que en la mayoría de las 
agresiones contra los padres no había relación directa entre el consumo de tóxicos y la conducta violenta. Los resultados sobre el abuso de sustancias no son consistentes, tal vez debido a las diferencias culturales, al tipo de muestra y a los diferentes aspectos del consumo de drogas evaluados (consumo regular de sustancias, abuso de sustancias, tendencia al consumo o consumo momentos antes de cometer la agresión), por lo que esta cuestión requeriría mayor investigación.

Las investigaciones realizadas hasta el momento acerca de la violencia filioparental no han llegado a resultados concluyentes sobre un perfil psicológico específico de los menores que agreden a sus padres. Así por ejemplo, en el estudio con muestra de la población general de Calvete et al. (2011) se halló que los jóvenes que agredían a sus padres se caracterizaban por tener una baja autoestima, así como creencias de grandiosidad y justificación de la violencia. Por su parte, Nock y Kazdin (2002), analizando una muestra clínica, encontraron un menor nivel de tolerancia a la frustración en estos jóvenes. En cuanto al perfil psicopatológico de los jóvenes violentos con sus padres, Nordstrom y Gunnar (2003) señalan que son pocos los adolescentes que sufriendo una enfermedad mental grave como esquizofrenia, trastorno bipolar $\mathrm{o}$ depresión mayor tiendan a agredir a sus padres. No obstante, en un reciente estudio en el que se compara una muestra judicial de jóvenes denunciados por violencia filioparental con otro tipo de infractores, se halló que los jóvenes que agredían a sus padres mostraban mayores tasas de hospitalizaciones psiquiátricas, de intentos de suicidio y de medicación psicotrópica (Kennedy, Edmonds, Dann y Burnett, 2010). En el contexto español, estudios basados en muestra clínica y judicial han confirmado que aproximadamente la mitad de los menores presenta algún trastorno psicológico, y la categoría diagnóstica más frecuente es el déficit de atención y comportamiento perturbador (Cuervo et al., 2008; Cuervo y Rechea, 2010; González-Álvarez et al., 2010).

Teniendo en cuenta que las investigaciones sobre violencia filio-parental apenas se han centrados en el estudio del perfil psicológico/psicopatológico de los menores infractores, en el presente estudio se plantearon dos grandes objetivos: 


\section{Objetivos e hipótesis}

- Comprobar hasta qué punto las características psicológicas y/o psicopatológicas de los menores infractores que habían sido denunciados por abuso físico y/o verbal a sus progenitores eran distintivas de este grupo de jóvenes o podrían encontrarse comúnmente entre los menores infractores.

- Obtener un modelo de regresión para la violencia filio-parental en base a características individuales de los menores infractores que ayude a detectar y a prevenir este tipo de problema familiar.

Teniendo en cuenta estos objetivos, se plantearon las siguientes hipótesis:

1) Respecto al contexto escolar, los menores denunciados por VF presentarían más dificultades escolares que los menores infractores sin denuncias de ese tipo (Howard y Rottem, 2008; Pagani et al., 2004).

2) Los menores denunciados por VF mostrarían un menor nivel de tolerancia a la frustración (Nock y Kazdin, 2002), menor autoestima y más conductas violentas en general (Calvete et al., 2011), que los menores infractores de delitos comunes.

3) En el grupo de jóvenes denunciados por VF, en comparación con otro tipo de infractores, se hallaría un mayor nivel de consumo regular de sustancias (Pagani et al., 2004).

4) Se esperaba encontrar más trastornos psicológicos entre los menores denunciados por VF que entre los menores del grupo de otras infracciones, y el trastorno por déficit de atención y comportamiento perturbador sería el trastorno más frecuente (Cuervo et al., 2008; Cuervo y Rechea, 2010; González-Álvarez et al., 2010).

\section{Método}

\section{Participantes}

Se analizaron 413 expedientes incoados en la Fiscalía de Menores de Bilbao (Comunidad Autónoma del País Vasco, España) durante los años 1999-2006 y correspondientes a 103 menores. Se estudiaron los datos de los adolescentes con edades comprendidas entre los 14 y los 18 años ( $\bar{X}=15,33), 85 \%$ de los cuales eran chicos y el $15 \%$ chicas. La muestra se dividió en tres grupos: un grupo de infractores denunciados exclusivamente por violencia filio-parental (VF) $(n=35)$, un grupo de menores que había cometido otro tipo de delitos (NoVF) $(n=35)$, y un tercer grupo formado por Revista Española de Investigación Criminológica

Artículo 6, Número 9 (2011) $\quad$ www.criminología.net

ISSN: $1696-9219$ 
menores denunciados no sólo por violencia filio-parental sino también por algún otro tipo de delito $(\mathrm{VF}+)(n=33)$. En la muestra se incluyeron todos los menores con alguna infracción por VF en el periodo de tiempo referido, mientras que los infractores del grupo NoVF, por su parte, fueron seleccionados al azar entre los menores con algún expediente por otro tipo de infracción. Se halló que en el 67\% de los casos de violencia filio-parental ésta se refería a violencia física y psicológica contra los progenitores, mientras que el $29 \%$ de las denuncias hacían referencia exclusivamente a maltrato físico y el 4\% únicamente a violencia psicológica.

\section{Procedimiento y diseño}

La información necesaria para realizar el presente estudio procedía de expedientes de estos menores, en los que se recogían todos sus procesos judiciales y las intervenciones profesionales llevadas a cabo con ellos. El estudio se realizó en base a informes redactados por la Policía, el Equipo Psicosocial Judicial y los Servicios Sociales de los Juzgados de Menores de Vizcaya (España). El Equipo Psicosocial Judicial se encarga de recopilar información sobre el perfil psicológico de los jóvenes y sus familias a través de entrevistas semi-estructuradas mantenidas con los adolescentes y sus padres utilizando un protocolo estandarizado para cada uno de ellos (menor y padres o tutores legales), en el que figuran una serie de apartados con preguntas específicas para facilitar la labor de los entrevistadores. Por ejemplo, en el apartado de contexto escolar del protocolo para padres constaban preguntas como las siguientes: ¿Cómo es su rendimiento en el colegio? ¿Y su actitud y comportamiento? ¿Cómo se relaciona con iguales y profesores?

Tras revisar la literatura científica sobre la violencia juvenil contra los padres, analizar los protocolos estandarizados del Equipo Psicosocial Judicial de Vizcaya y estudiar en profundidad algunos expedientes a modo de ejemplo del Juzgado de Menores, se establecieron las variables a seleccionar de los archivos. Estas variables se agruparon en cinco bloques principales: (a) Características del delito: víctima (padre, madre o ambos) y tipo de abuso contra la misma (físico, psicológico o ambos), (b) Características personales del menor: sexo, edad, número total de delitos, número delitos VF, (c) Dificultades escolares: adaptación escolar, dificultades de aprendizaje, conductas disruptivas en el aula y absentismo escolar, (d) Violencia fuera del hogar: 
violencia hacia sus compañeros y hacia otros adultos que no son los padres, y (e) Problemas psicológicos ${ }^{2}$ : variables emocionales (autoestima, nivel de tolerancia a la frustración y empatía), consumo regular de sustancias (alcohol, tabaco, hachís, cocaína y drogas de diseño), tratamiento psicológico individual antes de la primera denuncia y diagnóstico clínico según la información aportada por los padres. El análisis de datos se realizó con el paquete estadístico PASW Statistics 18.

\section{Resultados}

Los análisis correspondientes a las diferencias entre los tres grupos ( $\mathrm{VF}+, \mathrm{VF}$ y NoVF) de las variables evaluadas entorno a variables del contexto escolar, violencia fuera del hogar, problemas emocionales y trastornos psicológicos, se encuentran en la Tabla 1. El análisis de datos realizado fue la prueba de $\chi^{2}$ de Pearson por ser adecuada para las variables dicotómicas junto al coeficiente de contingencia.

\footnotetext{
2 Evaluación por parte de los miembros del Equipo Psicosocial Judicial a través de un protocolo estandarizado que disponían para tal fin.
} 


\section{Tabla 1.}

Variables de riesgo personales en función del grupo

\begin{tabular}{|c|c|c|c|c|c|c|c|c|}
\hline Variables personales de riesgo de los menores & $\begin{array}{c}\mathrm{VF}+ \\
(n=33) \\
\%\end{array}$ & $\begin{array}{c}\mathrm{VF} \\
(n=35) \\
\%\end{array}$ & $\begin{array}{c}\text { NoVF } \\
(n=35) \\
\%\end{array}$ & $N$ & $g l$ & $\chi^{2}$ & $p$ & $r$ \\
\hline \multicolumn{9}{|l|}{ Problemas en el contexto escolar } \\
\hline Problemas de adaptación & 78 & 64 & 11 & 88 & 2 & 29,58 & 0,001 & 0,50 \\
\hline Dificultades de aprendizaje & 69 & 61 & 7 & 88 & 2 & 26,07 & 0,001 & 0,48 \\
\hline Conductas disruptivas en el aula & 90 & 83 & 41 & 77 & 2 & 18,60 & 0,001 & 0,44 \\
\hline \multicolumn{9}{|l|}{ Violencia fuera del hogar } \\
\hline Violencia entre iguales & 77 & 73 & 46 & 70 & 2 & 6,78 & 0,03 & 0,30 \\
\hline Violencia hacia otros adultos (no padres) & 91 & 92 & 38 & 69 & 2 & 21,52 & 0,001 & 0,49 \\
\hline \multicolumn{9}{|l|}{ Problemas emocionales } \\
\hline Baja autoestima & 75 & 65 & 26 & 69 & 2 & 12,14 & 0,002 & 0,39 \\
\hline Baja capacidad de empatía & 83 & 88 & 59 & 71 & 2 & 6,40 & 0,04 & 0,29 \\
\hline \multicolumn{9}{|l|}{ Trastornos psicológicos } \\
\hline Tratamiento psicológico individual prejudicial & 68 & 77 & 22 & 74 & 2 & 17,75 & 0,001 & 0,44 \\
\hline Trastorno psicológico & 36 & 29 & 6 & 103 & 2 & 9,40 & 0,009 & 0,29 \\
\hline Consumo regular de sustancias legales e ilegales & 94 & 95 & 71 & 84 & 2 & 8,74 & 0,01 & 0,31 \\
\hline
\end{tabular}

Nota. $V F+$ : jóvenes denunciados por violencia filio-parental y otro tipo de delitos; $V F$ : jóvenes denunciados exclusivamente por violencia filio-parental; NoVF:

jóvenes denunciados por otros tipos de delitos; $r$ : Coeficiente de contingencia

Revista Española de Investigación Criminológica

Artículo 6, Número 9 (2011) Www.criminología.net

ISSN: 1696-9219 


\section{Problemas en el contexto escolar}

Entre los jóvenes denunciados por VF los problemas en el ámbito escolar se presentan en mayor proporción que en los jóvenes denunciados por otros delitos, siendo las diferencias significativas entre los tres grupos en cuanto a los problemas de adaptación, dificultades de aprendizaje, conductas disruptivas en el aula y absentismo escolar. Después de la transformación de las cuatro variables dicotómicas en una cuantitativa denominada "Dificultades escolares" se aplicó un ANOVA de un factor de acuerdo con el grupo de pertenencia $[F(2,66)=22,77, p<0,001]$. Según la prueba post-hoc de Tukey, en el grupo VF+ $(\bar{X}=3,2)$ y VF $(\bar{X}=2,9)$ el nivel de dificultades escolares era superior que en el grupo $\operatorname{NoVF}(\bar{X}=0,8)$.

\section{Violencia fuera del hogar}

Respecto a la violencia fuera del hogar, hay que señalar que había diferencias significativas teniendo en cuenta los tres grupos tanto para la violencia entre iguales como para la violencia hacia otros adultos que no sean los padres, siendo más habituales estas conductas en los dos grupos VF. Con la transformación de las dos variables dicotómicas en una nueva variable "Violencia fuera del hogar", se verificó que este tipo de violencia era menos frecuente en el grupo NoVF que en el grupo VF+ $[U(N=51)=$ $118,5, p<0,001]$ y $\operatorname{VF}[U(N=41)=49,50, p=0,002]$.

\section{Problemas emocionales}

Las variables emocionales eran ordinales originalmente pero se transformaron en dicotómicas tomando como referencia la mediana. Los resultados indicaban que en los grupos VF y VF+ había más adolescentes con baja autoestima y bajo nivel de tolerancia a la frustración y baja capacidad de empatía que en el grupo de jóvenes denunciados por otro tipo de delitos. Después de la transformación de las tres variables dicotómicas en una cuantitativa denominada "Problemas emocionales" se aplicó un ANOVA de un factor de acuerdo con el grupo de pertenencia $[F(2,53)=8,88, p<0,001]$. Según la prueba post-hoc de Tukey los grupos VF+ $(\bar{X}=2,4)$ y VF $(\bar{X}=2,5)$ presentaban más problemas emocionales que el grupo $\operatorname{NoVF}(\bar{X}=1,3)$. 


\section{Trastornos psicológicos}

Los menores que habían recibido tratamiento psicológico individual antes de la primera denuncia pertenecían en mayor medida a los grupos VF $(77 \%)$ y VF+ (68\%) que al grupo NoVF (22\%). Además, los menores de los grupos VF+ (36\%) y VF (29\%) presentaban un mayor porcentaje de trastornos psicológicos que los del grupo NoVF (6\%). En cuanto al tipo de trastorno psicológico ( $\sin$ trastorno, trastorno por déficit de atención y comportamiento perturbador, trastorno de personalidad, otros trastornos) las diferencias también eran significativas $\left[\chi^{2}(6, N=102)=14,79, p=0,02, r=0,36\right]$. Entre los que tienen algún trastorno psicológico las diferencias más importantes se centran en el trastorno por déficit de atención y comportamiento perturbador, siendo más frecuente en los grupos VF+ (33\%) y VF (17\%) que en el grupo NoVF (3\%). El 77\% de los participantes no presentaba ningún diagnóstico clínico teniendo en cuenta sus expedientes. Es posible que la mayoría de ellos no tuvieran trastorno psicológico alguno, porque los infractores que tenían algún trastorno psicológico presentaban más expedientes por VF $(\bar{X}=1,71), t(33,13)=-4,01, p<0,001$, más conductas violentas fuera del hogar $(\bar{X}=1,8), t(27,10)=-3,29, p=0,003$, y más problemas escolares $(\bar{X}=$ $3), t(46,35)=-2,24, p=0,02$, que los que no tenían un trastorno psicológico $(\bar{X}=0,77$, $\bar{X}=1,1$ y $\bar{X}=2$, respectivamente).

Los diagnósticos clínicos de los jóvenes con alguna infracción por VF $(n=22)$, se clasificaron en cuatro categorías teniendo en cuenta las clasificaciones del DSM-IVTR (APA, 2000) y del CIE-10 (Organización Mundial de la Salud, 1992): Trastorno por déficit de atención y comportamiento perturbador $(77 \%, n=17)$, Trastorno de personalidad $(14 \%, n=3)$, Trastorno de estado de ánimo $(4,5 \%, n=1)$ y Dependencia de sustancias $(4,5 \%, n=1)$. En la categoría Trastorno por déficit de atención y comportamiento perturbador el $76 \%$ tenía un diagnóstico de trastorno disocial, mientras que el $24 \%$ presentaba trastorno por déficit de atención. En la categoría Trastorno de personalidad se presentaron dos diagnósticos: trastorno límite de la personalidad (67\%) y trastorno esquizoide de personalidad (33\%).

Considerando a todos los jóvenes denunciados por VF como un sólo grupo, se aplicó la distribución Chi-cuadrado para verificar las diferencias entre las proporciones de los cuatro diagnósticos clínicos $\left[\chi^{2}(3, N=22)=32,54, p<0,001\right]$. Estos resultados indican que los menores infractores de VF con diagnóstico clínico tienen más 
probabilidad de ser diagnosticados de trastorno por déficit de atención y comportamiento perturbador que de trastorno de la personalidad u otro tipo de trastorno.

En lo relativo al consumo de sustancias, aunque se trataba de una conducta bastante generalizada (el $86 \%$ de todos los participantes consumía alguna sustancia de forma regular), se descubrió que los menores con delitos de violencia filio-parental eran más propensos al consumo de sustancias en general (legales e ilegales). No obstante, realizados los análisis para cada tipo de sustancia, no se encontraron diferencias significativas entre los tres grupos de menores infractores.

Después de la transformación de las tres variables dicotómicas en una cuantitativa denominada "Problemas psicológicos", se aplicó un ANOVA de un factor de acuerdo con el grupo de pertenencia $[F(2,59)=1,36, p<0,001]$. Según la prueba post-hoc de Tukey, en los grupos $\operatorname{VF}+(\bar{X}=2,08)$ y VF $(\bar{X}=2,35)$ el nivel de problemas psicológicos era superior que en el grupo $\operatorname{NoVF}(\bar{X}=1,08)$.

\section{Modelo de regresión para los delitos de violencia filio-parental}

En la Tabla 2 se incluyen los resultados de un modelo de regresión con dos predictores (dificultades escolares y violencia fuera del hogar) y la variable criterio violencia filio-parental (medida por el número de expedientes por VF) $[F(2,44)=$ $\left.16,35, p<0,001, R^{2}=0,40\right]$. Los dos predictores fueron significativos y el modelo explicaba el $40 \%$ de la varianza.

\section{Tabla 2.}

Modelo de regresión para delitos de violencia filio-parental

\begin{tabular}{lccccc}
\hline & \multicolumn{2}{c}{$\begin{array}{l}\text { Coeficientes no } \\
\text { estandarizados }\end{array}$} & $\begin{array}{c}\text { Coeficientes } \\
\text { tipificados }\end{array}$ & & \\
\hline Modelo & $B$ & Error típico & Beta & $t$ & Sig. \\
\hline Problemas en el contexto escolar & 0,22 & 0,11 & 0,31 & 2,02 & 0,05 \\
Violencia fuera del hogar & 0,56 & 0,22 & 0,41 & 2,55 & 0,01 \\
\hline
\end{tabular}

Variable dependiente: Número de expedientes VF 


\section{Discusión}

El primer objetivo de este estudio era identificar el perfil psicológico/psicopatológico de los menores denunciados por violencia filio-parental. Los jóvenes denunciados por violencia contra los progenitores presentaban más problemas escolares, más violencia fuera del hogar, más consumo de sustancias, más trastornos psicológicos y menor nivel de empatía, autoestima y tolerancia a la frustración que otros menores infractores.

La primera hipótesis fue confirmada, ya que los menores denunciados por VF presentaban más dificultades escolares que los menores infractores sin denuncias de ese tipo. Los resultados del presente estudio irían en la línea de los hallazgos de investigaciones previas que han destacado la relación entre la violencia filio-parental y las dificultades escolares (González-Álvarez et al., 2010; Howard y Rotterm, 2008). Del mismo modo, Pagani et al. $(2003,2004)$ verificaron que concretamente las conductas agresivas en el contexto escolar predecían las conductas agresivas de los adolescentes contra sus madres, mientras que Calvete et al. (2011) encontraron una asociación entre el absentismo escolar y la violencia filio-parental. Por lo tanto, parece que las dificultades para controlar las tendencias agresivas se manifiestan desde muy temprana edad también en el contexto escolar, y pueden culminar en conductas agresivas de mayor calado contra los progenitores.

Respecto al perfil psicológico de estos jóvenes, tal y como se había hipotetizado, los resultados indican que los agresores filio-parentales poseen una menor autoestima y menor nivel de tolerancia a la frustración en comparación con otro tipo de jóvenes infractores. Si bien la baja autoestima ha sido frecuentemente relacionada con las conductas violentas de los adolescentes en general (p.e., Ellickson y McGuigan, 2000; McCloskey y Lichter, 2003), resulta interesante destacar que este rasgo es aún más pronunciado en los jóvenes que delinquen por VF. En un estudio reciente de Calvete et al. (2011) sobre violencia filio-parental en el ámbito de la población general también encontraron que los jóvenes que agredían a sus padres se caracterizaban por tener una baja autoestima, un estilo impulsivo de resolución de conflictos y conducta delincuente. Además, se había comprobado que los jóvenes que agreden a sus padres muestran un apego más frágil hacia éstos (Agnew y Huguley, 1989; Paulson, Coombs y Landsverk 1990), y a su vez en las relaciones de apego inseguras con los progenitores los hijos se 
caracterizan por tener una baja autoestima y baja capacidad de empatía (Huntsinger y Luecken, 2003; Simons, Paternite y Shore, 2001). Como estas dos características (baja autoestima y baja capacidad de empatía) aparecen en el perfil de los jóvenes con delitos de VF, futuros estudios podrían analizar más en profundidad si efectivamente estos jóvenes presentan un tipo de apego inseguro hacia sus padres.

Según la tercera hipótesis, se esperaba que en el grupo de denunciados por VF se encontrara un mayor consumo de sustancias que en el grupo de otros infractores. Esta hipótesis se confirmó parcialmente, ya que los jóvenes del grupo mixto (VF+) consumían significativamente más sustancias de forma regular que el grupo de otros infractores, pero no había diferencias significativas entre el grupo VF y otros infractores (NoVF). Pagani et al. (2009), tampoco llegaron a resultados concluyentes sobre el papel del consumo de sustancias en la violencia filio-parental. En su estudio hallaron que el uso problemático de sustancias doblaba la probabilidad de que los adolescentes dirigieran comportamientos violentos tanto de índole física como verbal hacia sus padres varones. Sin embargo, en un estudio previo centrado en la violencia hacia las madres (Pagani et al., 2004) solamente encontraron una asociación predictiva significativa para la violencia verbal hacia ellas. El consumo de alcohol y drogas podría ser un factor agravante de la situación de conflicto familiar pero no necesariamente el causante del mismo.

De acuerdo con la cuarta hipótesis, los jóvenes denunciados por VF tenían una mayor probabilidad de ser diagnosticados de algún trastorno psicológico que los demás infractores $\mathrm{y}$, además, dichos menores habían recibido tratamiento psicológico individual antes de la primera denuncia en mayor proporción. San Juan y Ocáriz (2009) estudiaron una amplia muestra $(N=320)$ de la Comunidad Autónoma Vasca y encontraron que el $12 \%$ de los menores infractores por delitos comunes presentaba algún problema psicológico diagnosticado por el que estaba recibiendo tratamiento farmacológico o de psicoterapia. Este porcentaje es relativamente inferior al de los infractores por violencia filio-parental obtenido en este estudio $(\mathrm{VF}+=36 \%$ y VF $=$ $29 \%$ ), lo cual sería otro indicio más a favor de que los menores que han sido denunciados por VF presentan más trastornos psicológicos que los infractores por delitos comunes, de acuerdo con los resultados de las escasas investigaciones existentes sobre el tema (p.e., Kennedy et al., 2010). También se cumplió la predicción respecto a la mayor frecuencia del diagnóstico de déficit de atención y comportamiento 
perturbador encontrada entre estos jóvenes, de acuerdo con los resultados de estudios nacionales previos (Cuervo et al., 2008; Cuervo y Rechea, 2010; González-Álvarez et al., 2010).

El segundo objetivo de este estudio era obtener un modelo de regresión para la violencia filio-parental. Aunque todas las variables estudiadas correlacionaban con la violencia filio-parental, solamente los problemas escolares y la violencia fuera del hogar resultaron predictores válidos de este tipo de violencia familiar. Estos resultados son coherentes con los hallazgos de investigaciones previas, ya que señalan la relación entre los problemas en el contexto escolar y las conductas violentas hacia los padres (González-Álvarez et al., 2010; Pagani et al., 2003, 2004). Además, las conductas antisociales de los adolescentes también se han asociado con conductas violentas hacia las figuras de autoridad (Calvete et al., 2011; Jaureguizar e Ibabe, 2012). El hecho de que los agresores filio-parentales de este estudio mostraran más problemas escolares que otros menores infractores, así como más conductas violentas fuera del hogar (con iguales y otros adultos), estaba relacionado con el diagnóstico de algún trastorno psicológico. A su vez, esto podría explicar por qué un elevado porcentaje de estos infractores había recibido tratamiento psicológico individual antes de la primera denuncia por VF. Estos resultados son coherentes con los obtenidos por Kennedy et al. (2010) en una muestra judicial, ya que hallaron más problemas emocionales y de conducta así como más conductas violentas fuera del hogar en los infractores por VF que en otro tipo de infractores.

En conclusión, es obvio que las causas o factores que influyen sobre la violencia filio-parental son múltiples. Un adolescente que maltrata a sus padres puede hacerlo bien por su temperamento agresivo, por trastornos psicológicos que no han sido adecuadamente tratados, por los modelos de comportamientos violentos que se transmiten en la sociedad actual y por la "impunidad" que en ocasiones se observa en ellos, por la influencia negativa de los estilos educativos de sus padres o por la violencia que se ejerce dentro del sistema familiar (violencia de hijos a padres, violencia marital...), o, probablemente, por la interacción de éstos y muchos otros factores. El presente estudio ha podido arrojar más luz a este tipo de conductas violentas que aún no están suficientemente analizadas en la literatura científica, lo que podrá favorecer el diseño de intervenciones psicológicas individuales más adecuadas para los infractores por violencia filio-parental, en lugar de aplicar tratamientos que sigan el mismo patrón 
para todos los menores infractores. Así, será importante que las intervenciones psicológicas con estos menores tengan en cuenta que los infractores que son violentos hacia alguno de sus progenitores presentan más trastornos psicológicos que los infractores que no lo son, y tal vez por eso muestren más problemas en el ámbito escolar y más conductas violentas fuera del hogar. En futuras investigaciones, convendría estudiar la implicación de cada trastorno psicológico en la violencia filio-parental, y la interacción con otros factores de riesgo del ámbito familiar, ya que por sí misma la existencia de un trastorno psicológico no justificaría el comportamiento violento de hijos hacia padres.

\section{Limitaciones}

Una de las limitaciones de este estudio es que la evaluación de las variables psicológicas se ha obtenido a través de la valoración de los psicólogos del equipo psicosocial judicial a partir de entrevistas estandarizadas a padres y a menores, por lo que el equipo investigador no ha podido recoger información complementaria a través de más entrevistas o cuestionarios. Además, la información sobre el diagnóstico psicológico de los jóvenes se obtuvo a partir de la información aportada por los padres.

Agradecimientos. Esta investigación ha sido financiada por el Departamento de Justicia, Empleo y Seguridad Social del Gobierno Vasco. 


\section{Referencias}

Agnew, R., \& Huguley, S. (1989). Adolescent violence toward parents. Journal of Marriage and the Family, 51, 699-711.

APA, American Psychiatric Association (2000). Diagnostic and statistical manual of mental disorders. Washington: American Psychiatric Association.

Calvete, E., Orue, I., \& Sampedro, R. (2011). Violencia filio-parental en la adolescencia: Características ambientales y personales. Infancia y Aprendizaje, 34, 349-363.

Cottrell, B. (2001). Parent abuse: The Abuse of Parents by their Teenage Children. Canada: The family Violence Prevention Unit Health. Recuperado el 18 de octubre de 2011, desde http://www.phac-aspc.gc.ca/ncfvcnivf/pdfs/Abuse_E.pdf

Cottrell, B., \& Monk, P. (2004). Adolescent-to-Parent Abuse. A Qualitative Overview of Common Themes. Journal of Family Issues, 25, 1072-1095.

Cuervo, A. L., \& Rechea, C. (2010). Menores agresores en el ámbito familiar. Un estudio de casos. Revista de derecho penal y criminología, 3, 353-375.

Cuervo, A. L., Fernández, E., \& Rechea, C. (2008). Menores agresores en el hogar. Boletín Criminológico, 106, 1-4.

Dodge, K. A., \& Petit, G. S. (2003). A biopsychosocial model of the development of chronic conduct problems in adolescence. Developmental Psychology, 39, 349371.

Ellickson, P. I., \& McGuigan, K. A. (2000). Early predictors of adolescent violence. American Journal of Public Health, 90, 566-572.

Evans, E. D., \& Warren-Sohlberg, L. (1988). A pattern of analysis of adolescent abusive behaviour towards parents. Journal of Adolescent Research, 3, 201216.

González-Álvarez, M., Gesteira, C., Fernández-Arias, I., \& García-Vera, M.P. (2010). Adolescentes que agreden a sus padres. Un análisis descriptivo de los menores agresores. Psicopatología Clínica Legal y Forense, 10, 37-53.

Howard, J., \& Rottem, N. (2008). It all starts at home. Male adolescent violence to mothers. Monash, Australia: Inner South Community Health Service Inc and Child Abuse Research Australia, Monash University. 
Huntsinger, E. T., \& Luecken, L. J. (2003). Attachment relationships and health behaviour: the mediational role of self-esteem. Psychology and Health, 19, $515-526$.

Ibabe, I., \& Jaureguizar, J. (2011). ¿Hasta qué punto la violencia filio-parental es bidireccional? Anales de psicología, 27, 265-277.

Ibabe, I., Jaureguizar, J., \& Díaz, O. (2007). Violencia filio-parental conductas violentas de jóvenes hacia sus padres. Vitoria-Gasteiz: Servicio Central de Publicaciones del Gobierno Vasco.

Jaureguizar, J., \& Ibabe, I. (2012). Conductas violentas de los adolescentes hacia las figuras de autoridad: El papel mediador de las conductas antisociales. Revista de Psicología Social, 27, 7-24.

Kennedy, T. D., Edmonds, W. A., Dann, K. T. J., \& Burnett, K. F. (2010). The clinical and adaptive features of young offenders of child-parent violence. Journal of Family Violence, 25, 509-520.

Matey, P. (2011). Cuando los hijos maltratan a sus padres. Recuperado el 23 de octubre de 2011, desde http://www.elmundo.es/elmundosalud/2011/10/06/noticias/1317926422.html

McCloskey, L. A., \& Lichter, E. (2003). The contribution of marital violence to adolescent aggression across different relationships. Journal of Interpersonal Violence, 18, 390-412.

Nock, M. K., \& Kazdin, A. E. (2002). Parent-directed physical aggression by clinicreferred youths. Journal of Clinical Child Psychology, 31, 193-205.

Nordstrom, A. K., \& Gunnar, K. (2003). Victim relations and victim gender in violent crimes committed by offenders with schizophrenia. Social Psychiatry \& Psychiatric Epidemiology, 38, 326-330.

Organización Mundial de la Salud (OMS) (1992). International Statistical Classification of Diseases and Related Health Problems, Tenth Revision. Volume 1. Ginebra: Organización Mundial de la Salud.

Pagani, L. S., Larocque, D., Vitaro, F., \& Tremblay, R. E. (2003). Verbal and physical abuse toward mothers: The role of family configuration, environment, and doping strategies. Journal of Youth and Adolescence, 32, 215-223.

Pagani, L. S., Tremblay, R. E., Nagin, D., Zoccolillo, M., Vitaro, M., \& McDuff, P. (2004). Risk factor models for adolescent verbal and physical aggression 
toward mothers. International Journal of Behavioral Development, 28, 528537.

Pagani, L., Tremblay, R.E., Nagin, D., Zoccolillo, M., Vitaro, F., \& McDuff, P. (2009). Risk Factor Models for Adolescent Verbal and Physical Aggression Toward Fathers. Journal of Family Violence, 24, 173-182.

Paulson, M. J., Coombs, R. H., \& Landsverk, J. (1990). Youth who physically assault their parents. Journal of Family Violence, 5, 121-133.

San Juan, C., \& Ocáriz, E., (2009). Evaluación de la intervención educativa y análisis de la reincidencia en la Justicia de Menores en la CAPV. Vitoria-Gasteiz: Servicio Central de Publicaciones del Gobierno Vasco.

Simons K. J., Paternite C. E., \& Shore C. (2001). Quality of parent/adolescent attachment and aggression in young adolescents. Journal of Early Adolescence, 21, 182-203.

Walsh, J. A., \& Krienert, J. L. (2007). Child-parent violence: An empirical analysis of offender, victim, and event characteristics in a national sample of reported incidents. Journal of Family Violence, 22, 563-574. 


\section{Currículum de las autoras:}

Izaskun Ibabe es doctora en Psicología por la Universidad del País Vasco. Ha dirigido diversas investigaciones sobre violencia filio-parental $y$ ha participado en investigaciones sobre el testimonio de los testigos presenciales y las conductas adaptativas y desadaptativas de los escolares en la etapa infantil. En la actualidad es profesora titular de universidad en el Departamento de Psicología Social y Metodología de las Ciencias del Comportamiento de la Universidad del País Vasco. Sus principales áreas de interés son la delincuencia juvenil, conductas violentas y prosociales en la etapa adolescente, junto a la psicología del testimonio.

Joana Jaureguizar es doctora en Psicología por la Universidad del País Vasco, y ha trabajado como psicóloga en programas de apoyo y seguimiento a familias de acogida en el Centro de Estudios e Intervenciones Psicológicas Lauka, bajo contrato y dirección de la Diputación Foral de Guipúzcoa. En la actualidad es profesora adjunta en el Departamento de Psicología Evolutiva y de la Educación de la Universidad del País Vasco. Sus principales áreas de interés son el ajuste psicosocial de los adolescentes y sus conductas violentas y prosociales en el ámbito familiar y escolar, así como la depresión infantil en contextos educativos. 